\title{
Enhanced Artificial Neural Network Approach to Identify Specific Binary Pattern
}

\author{
Jitendra Singh Sengar \\ BVM College of Technology \& Management, \\ Gwalior, MP, India \\ jitendrasinghsengar@gmail.com
}

\begin{abstract}
The main focus of this paper is to design a Artificial Neural Network Modal to identify specific binary pattern. This pattern will work at binary level to recognize that the received data is authentic at low level, through this work we are able to design a specific binary pattern and it would apply directly to the input layer to check accuracy in data at binary mean at low level. In this paper we have four bit data and user may arrange it in his own specific pattern, then ANN Modal will train for the given pattern then it will apply to check the pattern is found and data is authentic.
\end{abstract}

Keywords: Artificial Neural Network, Binary Pattern Recognition, Learning or training Algorithms

\section{Introduction}

Neural networks have emerged as a field of study within AI and engineering via the collaborative efforts of engineers, physicists, mathematicians, computer scientists, and neuroscientists. Although the strands of research are many, there is a basic underlying focus on pattern recognition and pattern generation, embedded within an overall focus on network architectures. Many neural network methods can be viewed as generalizations of classical pattern-oriented techniques in statistics and the engineering areas of signal processing, system identification, optimization, and control theory.

A broader view of a neural network architecture involves treating the network as a statistical processor, characterized by making particular probabilistic assumptions about data. Patterns appearing on the input nodes or the output nodes of a network are viewed as samples from probability densities, and a network is viewed as a probabilistic model that assigns probabilities to patterns.

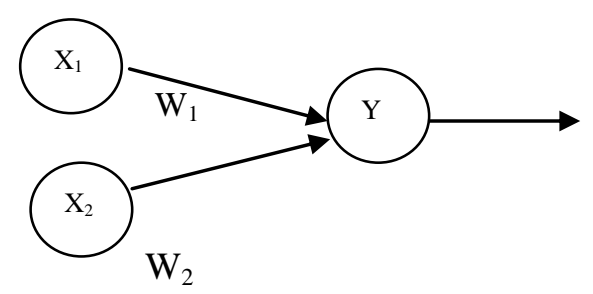

The figure shows a simple artificial neural net with two input neurons $\left(X_{1}\right.$, $X_{2}$ ) and one output neuron $(Y)$. The inter connected weights are given by $W_{1}$ and $\mathrm{W}_{2}$.

Other practical statistical issues that arise include the assignment of degrees of confidence to network outputs ("error bars"), the active choice of data points ("active learning"), and the choice between different networks. Progress has been made on all 
these issues by applying and developing statistical ideas. The statistical approach also helps in understanding the capabilities and limitations of network models and in extending their range.

\section{Learning Algorithms}

In most cases an ANN is an adaptive system that changes its structure based on external or internal information that flows through the network during the learning phase. It is a process in which the parameters of a neural network are adapted through a process of stimulation by the environment in which the network is embedded. The type of learning is determined by the manner in which the parameter changes take place.

Supervised learning is the machine learning task of inferring a function from supervised training data. The training data consist of a set of training examples. In supervised learning, each example is a pair consisting of an input object (typically a vector) and a desired output value (also called the supervisory signal). A supervised learning algorithm analyzes the training data and produces an inferred function, which is called a classifier (if the output is discrete) or a regression function (if the output is continuous). The inferred function should predict the correct output value for any valid input object. This requires the learning algorithm to generalize from the training data to unseen situations in a "reasonable" way (see inductive bias).

In supervised learning, we are given a set of example pairs $(x, y), x \in X, y \in Y$ and the aim is to find a function $f: X \rightarrow Y$ in the allowed class of functions that matches the examples. In other words, we wish to infer the mapping implied by the data; the cost function is related to the mismatch between our mapping and the data and it implicitly contains prior knowledge about the problem domain. Tasks that fall within the paradigm of supervised learning are pattern recognition (also known as classification) and regression (also known as function approximation).

Unsupervised learning - Most similar input vector is assigned to the same output unit. In this learning there is no external teacher to oversee the learning process. In other words, there are no specific samples of the function to be learned by the network. Rather, provision is made for a task-independent measure of the quality of representation that the network is required to learn and the free parameters of the network are optimized with respect to that measure. In unsupervised learning, We are given a training set $\left\{\mathbf{x}_{\mathbf{i}} ; i=1,2\right.$, ..., $m$ \}, of unlabeled vectors in $\mathrm{R}_{\mathrm{n}}$. The objective is to categorize or discover features or regularities in the training data. In some cases the $\mathbf{x}_{\mathrm{i}}$ must be mapped into a lower dimensional set of patterns such that any topological relations existing among the $\mathbf{x}_{\mathbf{i}}$ are preserved among the new set of patterns.

Reinforcement Training - Right answer is not provided but indication of whether 'right' or 'wrong' is provided. Inspired by behaviorist psychology, reinforcement learning is an area of machine learning in computer science, concerned with how an agent ought to take actions in an environment so as to maximize some notion of cumulative reward. The problem, due to its generality, is studied in many other disciplines, such as control theory, operations research, information theory, simulation-based optimization, statistics, and Genetic Algorithms. In the operations research and control literature the field where reinforcement learning methods are studied is called approximate dynamic programming.

\section{Pattern Analysis}

\section{A. Pattern Recognition}

Machine level recognition, description, classification, and grouping of patterns are important problems in a variety of engineering and scientific disciplines such as biology, psychology, medicine, marketing, computer vision, artificial intelligence, and remote sensing. A pattern could be a fingerprint image, a handwritten cursive word, a human 
face, or a speech signal. Given a pattern, its recognition/classification may consist of one of the following two tasks:

1) supervised classification (e.g., discriminant analysis) in which the input pattern is identified as a member of a predefined class,

2) unsupervised classification (e.g., clustering) in which the pattern is assigned to a hitherto unknown class.

The recognition problem here is being posed as a classification or categorization task, where the classes are either defined by the system designer (in supervised classification) or are learned based on the similarity of patterns (in unsupervised classification).

The design of a pattern recognition system essentially involves the following three aspects:

1) data acquisition and preprocessing.

2) data representation.

3) decision making.

The problem domain dictates the choice of sensor(s), preprocessing technique, representation scheme, and the decision making model. It is generally agreed that a welldefined and sufficiently constrained recognition problem (small interclass variations and large interclass variations) will lead to a compact pattern representation and a simple decision making strategy. Learning from a set of examples (training set) is an important and desired attribute of most pattern recognition systems. The four best known approaches for pattern recognition are: 1) template matching, 2) statistical classification, 3) syntactic or structural matching, and 4) neural networks.

\section{Proposed Algorithm}

The network starts by clamping the input at F1. Because the output of F2 is zero, G1 and $\mathrm{G} 2$ are both on and the output of $\mathrm{F} 1$ matches its input. The pattern is sent to F2, and in F2 one neuron becomes active. If there is a substantial mismatch between the two patterns, the reset signal will inhibit the neuron in F2 and the process is repeated. To obtain a set of weights $\mathrm{w}_{\mathrm{ij}}$ from a set of training pattern pairs $\{\mathrm{s}: \mathrm{t}\}$ such that when $\mathrm{s}$ is applied to the input layer, $t$ is computed at the output layer, for all training pairs $s: t, t_{j}=$ $f\left(s^{T} w_{j}\right)$ for all $j$.

1. Initialization:

$$
\begin{aligned}
& w_{j i}^{b}(0)=1 \\
& w_{i j}^{f}(0)=\frac{1}{1+N}
\end{aligned}
$$

Where, $\mathrm{N}$ is the number of neurons in $\mathrm{F} 1, \mathrm{M}$ the number of neurons in $\mathrm{F} 2$.

2. Apply Binary input pattern $x$

3. Summing weighted input to output units:

$$
y_{-} i n_{j}=\sum_{i} x_{i} w_{i j}
$$


4. Apply activation function: threshold

$$
y_{j}=\left\{\begin{array}{lll}
1 & \text { if } & y_{-} i n_{j}>0 \\
0 & \text { if } & y_{-} i n_{j} \leq 0
\end{array}\right.
$$

5. Weights are computed by Hebbian rule of all training pairs.

$$
W=\sum^{P} s_{i}^{T}(p) t_{j}(p)
$$

6. select the winhtintg neuron $\mathrm{k}(0 \leq \mathrm{k}<\mathrm{M})$ :

7. vigilance test: if

$$
\frac{\boldsymbol{w}_{k}^{b}(t) \cdot \boldsymbol{x}}{\boldsymbol{x} \cdot \boldsymbol{x}}>\rho,
$$

where. denotes inner product, vigilance threshold $\rho$,

Note that, the inner product, which will be large if pattern are near to each other.

\section{Result And Analysis}

In this paper, we have taken following training samples

$\mathrm{s}(\mathrm{p})$

$\mathrm{p}=01$
$\mathrm{p}=02$
$\mathrm{p}=03$
$\mathrm{p}=04$
$\mathrm{p}=05$$\quad \begin{aligned} & \left(\begin{array}{llll}0 & 0 & 0 & 0\end{array}\right) \\ & \mathrm{l}\end{aligned}$

So we assume that have only two pattern for recognition like (0010) and (0111) by using w then.

$$
\mathrm{s}(\mathrm{p}) \quad \mathrm{R}(\mathrm{p})
$$

$\mathrm{p}=1 \quad\left(\begin{array}{llll}0 & 0 & 0 & 0\end{array}\right)$ 


\begin{tabular}{|c|c|}
\hline$=2$ & $\left(\begin{array}{lllll}0 & 0 & 1 & 0\end{array}\right)$ \\
\hline$p=3$ & $\left(\begin{array}{llll}0 & 0 & 0 & 1\end{array}\right)$ \\
\hline$p=4$ & $\left(\begin{array}{llll}0 & 1 & 1 & 0\end{array}\right)$ \\
\hline$p=5$ & $\left(\begin{array}{llll}1 & 0 & 0 & 1\end{array}\right)$ \\
\hline$p=6$ & 10 \\
\hline$=7$ & $\left(\begin{array}{ll}0 & 1\end{array}\right.$ \\
\hline & \\
\hline
\end{tabular}

r2

r7

In this step, test the whole network then recognize all input vector and getting the accurate result with respect to (0010) r2 and (0111)r7 patterns with less execution time.

\section{Conclusion and Future Work}

I have reviewed pattern classification and considered a range of computational models that incorporate learning, and describe experimental studies consistent with the idea, which seems to follow from a approach, that there are processes at work strengthening the response a person makes to an input, either when this is recognized or not recognized. learning can provide a basis for thinking about how we may learn from our own responses to things, in the absence of external teaching information.

\section{References}

[1] Syed Ayaz Ali Shah, Azzam ul Asar and S.F. Shaukat, "Neural Network Solution for Secure Interactive Voice Response", World Applied Sciences Journal 6 (9), 1264-1269, 2009.

[2] Xinyu Guo, Xun Liang and Xiang Li, "A Stock Pattern Recognition Algorithm Based on Neural Networks", Third International Conference on Natural Computation, Volume 02,2007

[3] Wenjin Dai and Ping Wang, "Application of Pattern Recognition and Artificial Neural Network to Load Forecasting in Electric Power System", Third International Conference on Natural Computation, Volume 01,2007.

[4] Lin He, Wensheng Hou, Xiaolin Zhen and Chenglin Peng, "Recognition of ECG Patterns Using Artificial Neural Network", Sixth International Conference on Intelligent Systems Design and Applications, Volume 02,2006,

[5] Nallasamy Mani and Bala Srinivasan, "Application of Artificial Neural Network Model for Optical Character Recognition", IEEE international conference, 12-15 Oct, 1997.

[6] A.K. Jain, J. Mao, and K.M. Mohiuddin, "Artificial Neural Networks: A Tutorial”, Computer, pp. 3144, Mar, 1996.

[7] Young-Sang Han, Seong-Sik Min, Won-Ho Choi and Kyu-Bock Cho, "A Learning Pattern Recognition System using Neural Network for Diagnosis and Monitoring of Aging of Electrical Motor", International Conference, 9-13 Nov, 1992. 
International Journal of Hybrid Information Technology Vol.8, No.6 (2015) 